\title{
From search to social: Innovation for today's researchers
}

\author{
Christine McCue \\ Vice President, Marketing, Chemical Abstracts Service, Columbus, OH, USA
}

As anyone who owns a computer or even a smart phone understands, social media have the power to transform how people connect to and exchange information. And the influence is growing, as people of nearly every demographic - from teens to Baby Boomers, from academics to business professionals take advantage of what social media have to offer.

Social media accounted for $11 \%$ of all time spent online in the US in 2009, according to comScore. As reported by Nielsen, that time will increase by 82\% from December 2009 to December 2010.

As communication shifts rapidly in favor of online forms, companies like Chemical Abstracts Service (CAS) are eager to participate in order to deliver on the needs of their information users.

Since its inception, CAS has been synonymous with innovation. For more than a century, the company has been an industry pioneer, adopting the newest technologies, meeting the needs of its customers thoughtfully and thoroughly, and developing new offerings that deliver exceptional service.

\section{A company born in innovation}

Founded in 1907 to help scientists benefit from the published work of their colleagues, Columbusbased CAS has grown into a global publishing organization committed to serving those on the front lines of scientific discovery - researchers.

Each day, hundreds of scientists at CAS' 50 -acre Columbus campus and around the globe build and maintain the world's most comprehensive collections of information about chemistry and related science. Researchers from Fortune 500 corporations and universities world-wide access and use CAS' information to develop everything from more environmentally friendly fuels to smarter and faster computers, from healthier foods to drugs with fewer side-effects.

In addition to compiling the most authoritative databases of their kind, CAS technologists build innovative search tools like STN ${ }^{\circledR}$ and SciFinder ${ }^{\circledR}$ that enable researchers to explore CAS' extensive content easily. More than 10,000 scientific journals, patents, and other reputable sources in CAS databases are coupled with sophisticated search-and-analysis tools to bring speed, accuracy, and cutting-edge relevance to customers' scientific research.

Together, more than $1300 \mathrm{PhD}$ scientists, IT professionals, and other staff at CAS work continuously to develop innovative solutions for advancing global research. 


\section{Evolution of science and search}

Since CAS was founded, the boundaries between scientific disciplines have slowly dissolved, leaving more researchers working on new fronts. This interdisciplinary environment presented CAS with an opportunity: to provide pertinent information to scientists who found themselves entrenched within new disciplines and working outside the boundaries of their knowledge.

Throughout its history, CAS has helped scientists worldwide to collaborate and benefit from each other's published work. To continue this effort in the 21st century, CAS keeps listening intently to its customers' needs while staying abreast of changing technology.

\section{A lesson in mobile}

CAS' earliest foray into interactive technology was in the mobile space.

First introduced to market in 1999, the BlackBerry did not gain traction until 2004, with about 2 million users. At this time CAS noted the growing interest in and availability of mobile communications and began to explore the potential of a mobile application.

Recognizing that SciFinder search functions could be incorporated into the still-novel and developing mobile technology of the time, CAS launched a pilot mobile application in April 2005 at the CAS European Conference in Vienna. This basic application, CAS Mobile ${ }^{\mathrm{TM}}$, was designed to enable mobile scientific searching (Fig. 1).

Yet CAS quickly found that, while many executives owned a BlackBerry in 2005, most researchers and chemists did not - and those who did were not readily using their device to conduct scientific research.

CAS was ahead of its time when it launched CAS Mobile, and received praise among third parties for its innovation, including editorial reviews in publications like Chemical \& Engineering News. An on-camera appearance of a mobile chemical search in a 2008 episode of CSI: New York demonstrated a practical need for mobile access to verified chemical information.

Still, the company learned a valuable lesson: one cannot innovate beyond its users' demands.

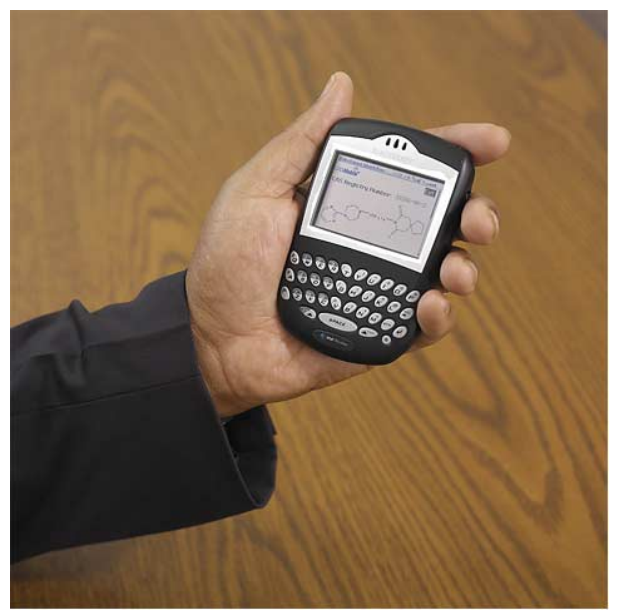

Fig. 1. CAS Mobile accessed on a Blackberry mobile device. 


\section{Search on every device}

Today is a different story for mobile. With more than 45 million smart phone users in the US, or 19\% of the country, more and more customers are coming to expect access on-the-go. According to a recent CAS survey of 380 customers, two-thirds (66\%) of its users own a mobile device and more than half (54\%) use this device for work-related research. This number is even higher, 57\%, among academics.

The CAS survey further demonstrated that customers are using or would use their mobile devices to investigate a variety of areas including conducting searches on general topics $(86 \%)$, receiving alerts to stay current (47\%), looking up authors' and investors' research (36\%) and obtaining competitive intelligence (14\%).

Clearly, most current CAS customers can access information on a mobile device, and in fact are already doing so (Fig. 2). They also would like to - or already are - conducting many different types of searches on-the-go. This is a vast change from the lackluster 2005 environment when CAS Mobile was launched. It demonstrates that, often, innovation not yet demanded by users will one day soon be in demand.

CAS also discovered that its users want to access SciFinder on a variety of devices, including the iPhone $(45 \%)$, iPod or similar media players $(16 \%)$, BlackBerry $(13 \%)$, handheld PC $(8 \%)$, or Trio $(1 \%)$. The new challenge for CAS is not to offer a mobile platform but to provide an application for each device, responding to its customers' requests.

In 2009, CAS developed an iPhone application that made CAS' Colors of Chemistry calendar content (http://cascolors.compexinc.com) available to customers and the public alike (Fig. 3).

CAS will continue to evaluate the findings of its customer research to determine the best course for future mobile application development.

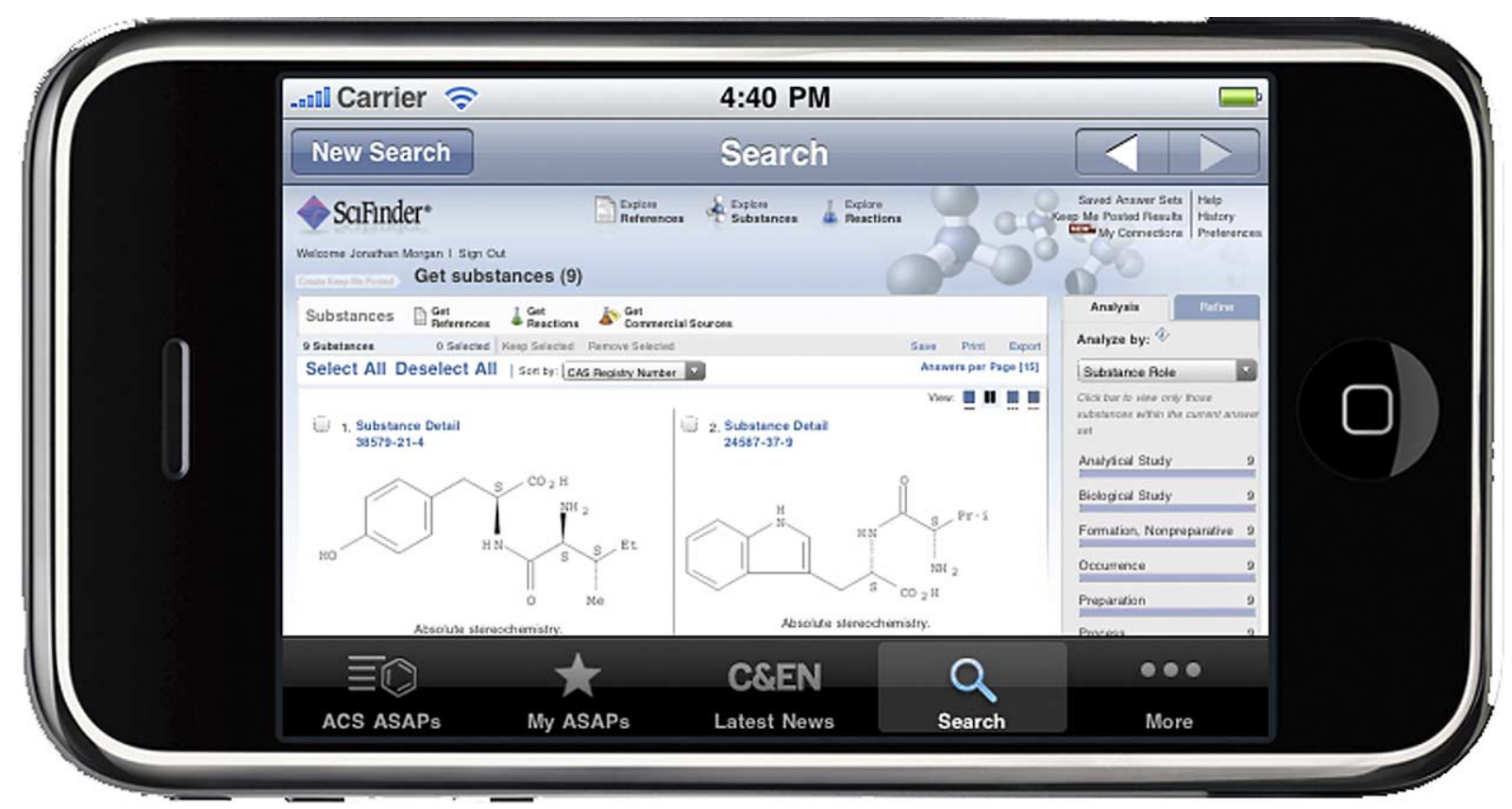

Fig. 2. A SciFinder substance search conducted on an iPhone. 


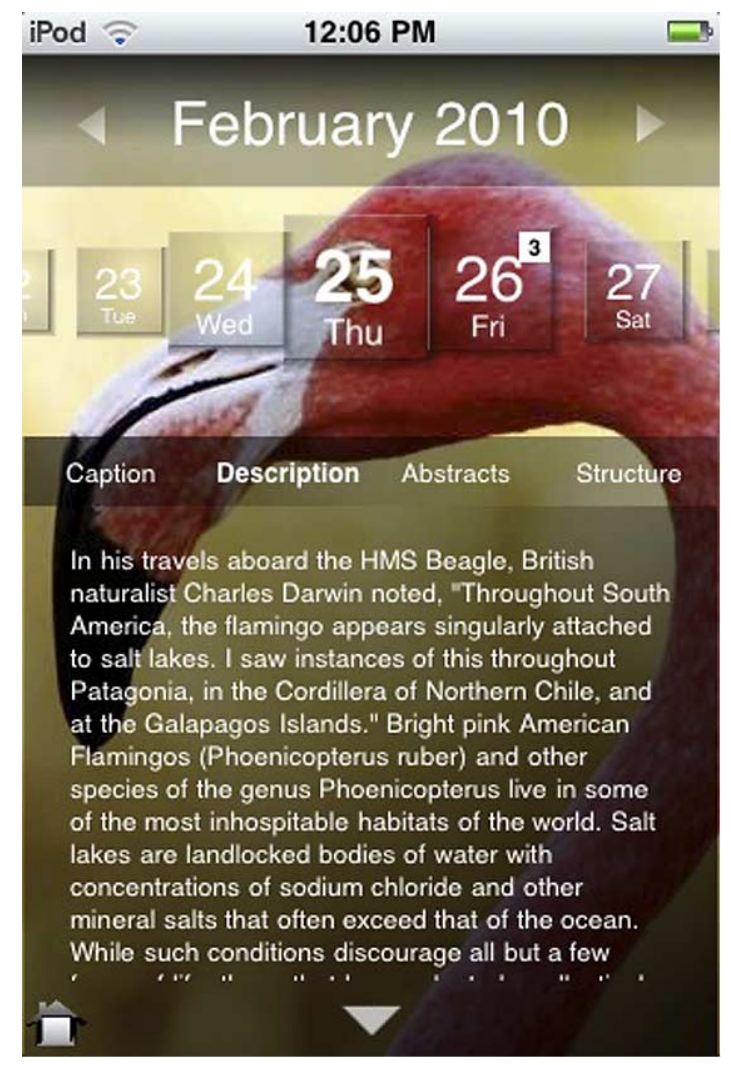

Fig. 3. The CAS colors of chemistry calendar accessed on an iPod.

\section{Search becomes social}

Even while embracing social media, at its heart CAS has remained the same.

Not long after developing CAS Mobile, CAS researchers became increasingly aware of the new ways in which chemists and other scientific professionals were sharing information online. Starting with older outlets like e-mail discussion lists and evolving to newer platforms such as Twitter, CAS found that its customers were often discussing their experiences in and outside the lab via social media. They were connecting professionally using tools such as LinkedIn and Facebook, and publishing ideas and commenting on each others' evolving thoughts via blogs, wikis, and other tools.

Understanding that its customers were already using these networks, CAS was eager to find where, why, and what they talked about. It discovered that an overwhelming 94\% of customers used a Facebook account, while others were on LinkedIn and Twitter, among a few other sites. Of these users, nearly half used the sites for both personal and professional use. Commercial/corporate, government, and non-profit customers were even more fully engaged (58\%).

Appreciating its customers' desire for an interactive community, CAS created Facebook and LinkedIn accounts for SciFinder, Twitter accounts for CAS and STN, and a YouTube channel for the brand. But, the company wondered, was there an appetite among its users for a unique social networking site?

When asked, more than two-thirds said they would join a SciFinder-related forum or group if one was available and said that they would do so in order to collaborate with others performing similar searches, troubleshoot specific problems, and gain support from CAS. 
Thus the company took its commitment to collaboration to the next level by directly incorporating social elements into SciFinder. Now in a beta version, the features are meant to be "social media light", mimicking the social networking experience users are already accustomed to online. The features are flexible and open and can accommodate both experienced and inexperienced social media users.

Using the new SciFinder collaboration tools, users can connect with others within their own research site or by inviting or accepting invitations to connect from SciFinder users they know, share research content with their groups, share information via reference tags, tag user-created labels, and comment on any user's content.

\section{Building from the basics}

Throughout its 100-year history, CAS has worked carefully and thoughtfully to build solutions that are founded in research. This research includes listening to its customers at every turn and developing new resources or tools to meet their changing demands.

CAS has long taken note of technological trends and carefully evaluated when to leverage them in developing solutions that are useful and appropriate for its customers.

CAS is focused on delivering value to customers and on making access to its robust and unrivaled data as simple and convenient as possible. This is true innovation. 